\title{
JAFFAL: Detecting fusion genes with long read transcriptome sequencing
}

Nadia M. Davidson ${ }^{1,2 \dagger}$, Ying Chen ${ }^{3}$, Georgina L. Ryland ${ }^{1,4,5}$, Piers Blombery ${ }^{1,5}$, Jonathan Göke ${ }^{3,6}$, Alicia Oshlack ${ }^{1,2,5 \dagger}$

${ }^{1}$ Peter MacCallum Cancer Centre, Victoria, Australia

${ }^{2}$ School of BioSciences, University of Melbourne, Victoria, Australia

${ }^{3}$ Genome Institute of Singapore, Singapore, Singapore

${ }^{4}$ Centre for Cancer Research, University of Melbourne, Victoria, Australia

${ }^{5}$ Sir Peter MacCallum Department of Oncology, University of Melbourne, Victoria, Australia

${ }^{6}$ National Cancer Centre Singapore, Singapore, Singapore

†Corresponding authors: nadia.davidson@petermac.org and alicia.oshlack@petermac.org

\begin{abstract}
Massively parallel short read transcriptome sequencing has greatly expanded our knowledge of fusion genes which are drivers of tumor initiation and progression. In cancer, many fusions are also important diagnostic markers and targets for therapy. Long read transcriptome sequencing allows the full length of fusion transcripts to be discovered, however, this data has a high rate of errors and fusion finding algorithms designed for short reads do not work. While numerous fusion finding algorithms now exist for short read RNA sequencing data, methods to detect fusions using third generation or long read sequencing data are lacking.
\end{abstract}

Here we present JAFFAL a method to identify fusions from long-read transcriptome sequencing. We validated JAFFAL using simulation, cell line and patient data from Nanopore and PacBio. We show that fusions can be accurately detected in long read data with JAFFAL, providing better accuracy than other long read fusion finders and within the range of a state-of-the-art method applied to short read data. By comparing Nanopore transcriptome sequencing protocols we find that numerous chimeric molecules are generated during cDNA library preparation that are absent when RNA is sequenced directly. Finally, we demonstrate that JAFFAL enables fusions to be detected at the level of individual cells, when applied to long read single cell sequencing. JAFFAL is open source and available as part of the JAFFA package at https://github.com/Oshlack/JAFFA/wiki.

Keywords: fusions, translocations, RNA sequencing, long reads, nanopore, pacbio 


\section{Background}

Genomic rearrangements are common in the landscape of cancer and when breakpoints occur within different genes these can be transcribed into a new hybrid transcript, producing a so-called fusion gene. Fusions may drive cancer through activation of onocogenes [1] or inactivation of tumour suppressors. Often such fusions are recurrent across patient cohorts and novel drugs have been developed to specifically target a number of them [2]. Fusion detection can therefore inform cancer care, and eliciting their function in cancer initiation and progression is an ongoing area of research.

Over the last decade, massively parallel short read transcriptome sequencing has greatly expanded our knowledge of fusion genes across cancers and is increasingly being used for clinical diagnostics [3-5]. For example, The Cancer Genome Atlas (TCGA) utilised short read transcriptome sequencing across a range of tumour types to estimate that approximately $16 \%$ of cancers have a fusion event which drives the disease [6]. Fusion discovery through sequencing has necessitated the development of dedicated bioinformatics methods. Since the advent of the first approaches [7,8], fusion finding has improved in both accuracy and speed, and there are now numerous tools available [9-12].

Third generation, or long read sequencing technologies, as offered by Oxford Nanopore Technologies (ONT) [13] and Pacific Bioscience (PacBio) [14], can provide novel insight into fusions and their role in cancer. Unlike short read sequencing, long read sequencing does not require fragmentation, hence the full length of individual mRNA molecules can be sequenced. Long range information about the structure and sequence of fusion transcripts, including splicing and variants, away from the breakpoint is retained. Long read sequencing has several other advantages, for example ONT allows RNA to be sequenced directly, without reverse transcription and therefore RNA modifications can be measured [15]. In addition, rapid and remote diagnostics may be possible with ultra portable sequencing machines and rapid workflows $[16,17]$. Finally, new protocols allow full length sequencing of genes at the level of single cells [18-20].

Most fusion finders rely on short read alignment algorithms, which are incapable of accurately and efficiently mapping long reads [21]. An additional challenge is that the raw data generated by third generation technologies have a high rate of errors [22], in particular insertion and 
deletions, that short read algorithms were not designed to account for. As a result, to the best of our knowledge, only three fusion finding methods are available for long read transcriptome data: JAFFA [23] is a pipeline we previously developed and although it can process transcriptome sequencing data of any length, it has low sensitivity when error rates are high; Aeron [24] detects fusions by aligning long reads to a graph based representation of the reference transcriptome; and LongGF [25] analyses genome mapped long read data and detects fusions by identifying reads aligning to multiple genes.

To take advantage of new long read sequencing technologies for fusion finding and characterisation, we have developed JAFFAL, a new method which is built on the concepts developed in JAFFA and overcomes the high error rate in long read transcriptome data by using alignment methods and filtering heuristics which are designed to handle noisy long reads. We validated JAFFAL using simulated data as well as cancer and healthy cell line data for ONT and PacBio. By comparing ONT transcriptome sequencing protocols we show that numerous chimeric molecules are generated during cDNA library preparation that are absent when RNA is sequenced directly. JAFFAL effectively filtered these events by accurately determining break-point positions relative to exon boundaries. We show JAFFAL is the most accurate fusion finder available for noisy long read data, allowing fusions to be detected in long read data with similar accuracy as short reads. On two patient ONT sequencing samples, JAFFAL was able to detect clinically relevant fusions. Finally, as a proof-of-feasibility, we apply JAFFAL to long read single cell sequencing of five cancer cell lines and demonstrate its ability to recover known fusions at the level of individual cells. JAFFAL is open source and available as part of the fusion finding package JAFFA, versions 2.0 and higher (https://github.com/Oshlack/JAFFA/wiki).

\section{Results and Discussion}

\section{JAFFAL pipeline}

JAFFAL is a new multistage pipeline (Figure 1) written in bpipe [26] and was motivated by our approach from the Direct mode of JAFFA [23]. The pipeline consists of the following steps: (1) Fusions are detected by first aligning long reads to a reference transcriptome (hg38 gencode version 22) [27] using the noise tolerant long read aligner minimap2 [28]. (2) Reads consistent with a fusion gene, ie. those with sections aligning to different genes, are selected for further 
analysis and (3) subsequently aligned to the reference genome hg38, also using minimap2. Reads which do not span multiple genes after reference genome alignment are removed. This double alignment, to a reference transcriptome and genome, ensures that false positives are minimised, and reduces computational time, as only a small subset of reads need to be aligned to the full reference genome.

Next, (4) JAFFAL uses the end position of reference genome alignments to determine fusion breakpoints. Due to the high error rate in long read sequencing, alignment end positions may be inaccurate. To account for this, JAFFAL employs a strategy which anchors transcript breakpoints to exon boundaries. While structural rearrangements commonly occur within introns, splice sites are usually preserved, creating fusion transcripts where the breakpoint in the RNA is at the end or start of an exon. JAFFAL will realign breakpoints to the exon boundaries if exon boundaries are identified within $20 \mathrm{bp}$ of the original alignment breakpoints. This is only done if the adjustments on the 5' and 3' sides of the break are consistent with one another, and result in a new breakpoint at exon boundaries for both the 5' and 3' gene. All such exon boundary breakpoints will be reported by JAFFAL.

Due to insertion and deletion errors, or genuine breakpoints within an exon body, many reads will not satisfy the requirements for breakpoint adjustment. These reads are clustered by genomic position. One breakpoint is reported for each cluster, which will be either the one preserving exon boundaries, or the one with the highest read support. Clustering is achieved by iterating through all non-exon boundary breakpoints, starting with the one with the least read support. The breakpoint's reads will be reassigned to the closest breakpoint from other reads within $50 \mathrm{bp}$ (euclidean genomic distance). If no other breakpoint is found within 50bp the breakpoint is reported.

Finally, (5) breakpoints are ranked into "High Confidence", "Low Confidence" and "Potential Trans-Splicing" classes (Figure 1), similar in concept to the ranking in JAFFA for short reads [23]. "High Confidence" fusions are supported by two or more reads with breakpoints aligning to exon boundaries. "Low Confidence" fusions are also supported by two or more reads, but breakpoints do not align to exon boundaries. "Potential Trans-Splicing" events are supported by a single read, with breakpoints aligning to exon boundaries (Figure 1). Numerous "Potential Trans-Splicing" events are seen in healthy RNA-Seq samples [23,29], and should generally be filtered out. However some true fusions may be reported as "Potential Tran-Splicing", for 
example those with low expression levels or in samples with low tumour purity. All other events are removed. Run-through transcription, identified by breakpoints within $200 \mathrm{kbp}$ of each other and where the genes are transcribed in the same order as the reference genome, are also filtered out by default, as are fusions which involve the mitochondrial chromosome. However, these events may be recovered by the user if needed.

For each breakpoint which passes filtering, JAFFAL reports the genes involved, genomic coordinates, number of reads supporting the event, ranking class, whether it is inframe and whether it has been seen before in the Mitelman database of genomic rearrangements [30]. Within each class, breakpoints are ranked by the number of supporting reads.

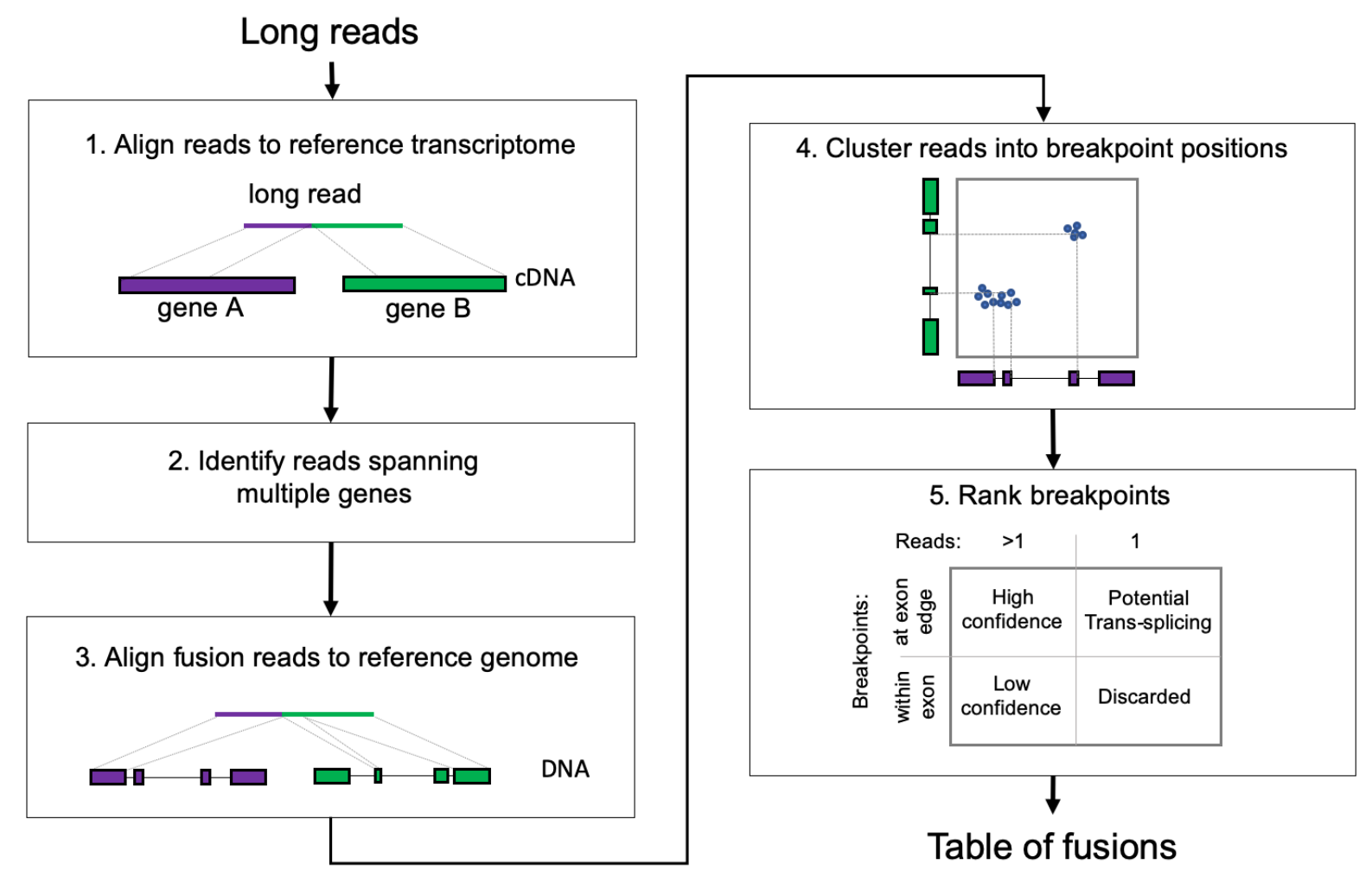

Figure 1. JAFFAL pipeline steps for fusion detection. Reads are aligned to the reference transcriptome, reads split across different genes are identified as candidate fusion reads and subsequently aligned to the reference genome for confirmation. Reads are clustered into breakpoint positions which are then ranked and reported (see text for details). 


\section{Simulated fusions are accurately detected in noisy long read data with JAFFAL}

JAFFAL's ability to detect fusions was tested on simulated data for the same 2500 fusion events simulated by Haas et al. [10]. For each fusion, Hass et al. selected two protein-coding genes at random. The breakpoint within each fusion was decided by joining a randomly selected exon from each gene, requiring a minimum $100 \mathrm{bp}$ of sequence from each. We simulated long reads from the resulting fusion gene sequences using Badread version 0.1.5 [31], which uses a noise model based on real data. The 2500 fusions were divided into 25 groups with varying coverage and read identity levels. Specifically, 500 fusion events were simulated across 5 coverage levels: $x 1, x 2, x 10, x 50$ and x100 reads. For each coverage we simulated 100 fusions each with a mean read identity of $75 \%, 80 \%, 85 \%, 90 \%$ and $95 \%$ (standard deviation $5 \%$ ). These read identities were designed to cover the range expected in real data. For example, the cell line data used to validate JAFFAL was estimated to have read identities in the range 80 to $85 \%$ for ONT and $85 \%$ to over $95 \%$ for PacBio (Supplementary Figure 1). No background reads from unfused genes were simulated (see Methods for more detail). ONT and PacBio data were simulated using the same fusion sequence, coverage level and read identity parameters.

For both ONT and PacBio, JAFFAL detected $98 \%$ of simulated fusions when the read identity was $90 \%$ or above and the coverage was 10 or greater (Figure $2 \mathrm{~A}$, Supplementary Figure 2A). Across all simulated fusions that were detected, approximately $84 \%$ were classed as high confidence. As expected, with low coverage and read identity, fewer fusions were detected. High error rates also impacted the fraction of supporting reads identified by JAFFAL. Amongst the fusions detected, the reported supporting reads were only $14 \%$ of the simulated coverage when the identity was $75 \%$, compared to $79 \%$ of coverage when the identity was $95 \%$. Most reads which failed to be reported did not align to two genes in the initial reference transcriptome mapping. This impacted up to $84 \%$ of simulated reads when the read identity was $75 \%$, with $40 \%$ failing to align to even one gene. The number of reads lost in other stages of the JAFFAL pipeline remained low, approximately $10 \%$, across all scenarios (Supplementary Figure 3 ).

JAFFAL's sensitivity on the simulated data was comparable to the alternative long read fusion finder, LongGF's (Figure 2B, Supplementary Figure 2B). As we were unsuccessful in running Aeron, results for that program are not shown. JAFFAL detected more fusions than LongGF when LongGF was run with default parameters ( $>1$ read support), but fewer when LongGF was allowed to report fusions with just one read support. This intermediate behaviour of JAFFAL is 
consistent with its reporting fusions with one read support conditional on the breakpoint coinciding with exon boundaries. At low read identities, this condition is more likely to fail due to poor alignment. JAFFAL was found to have superior breakpoint resolution to LongGF; for $96 \%$ of fusions detected by JAFFAL, the exact breakpoint was reported, compared to just $2 \%$ from LongGF. However, almost all breakpoints were within 20bp of the simulated position for both tools.

A

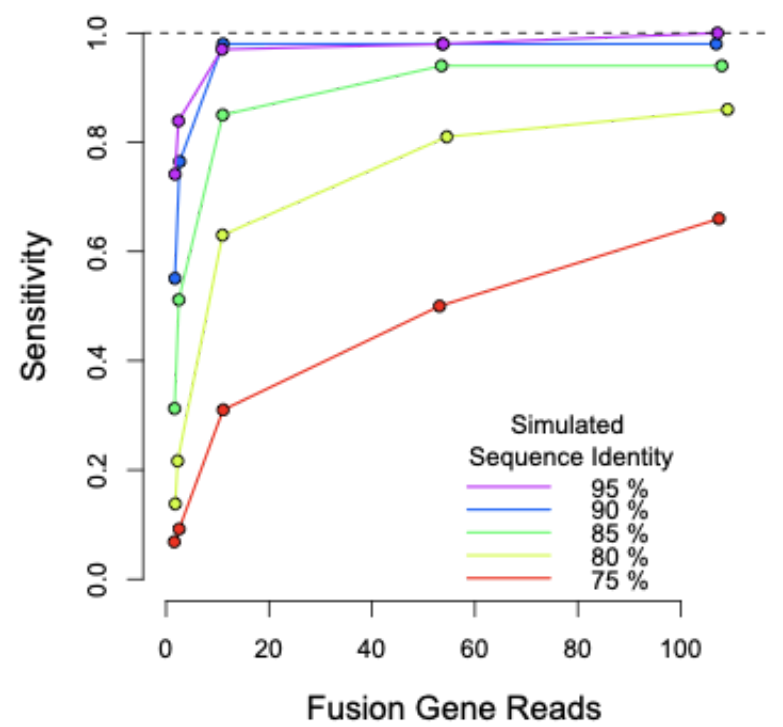

B

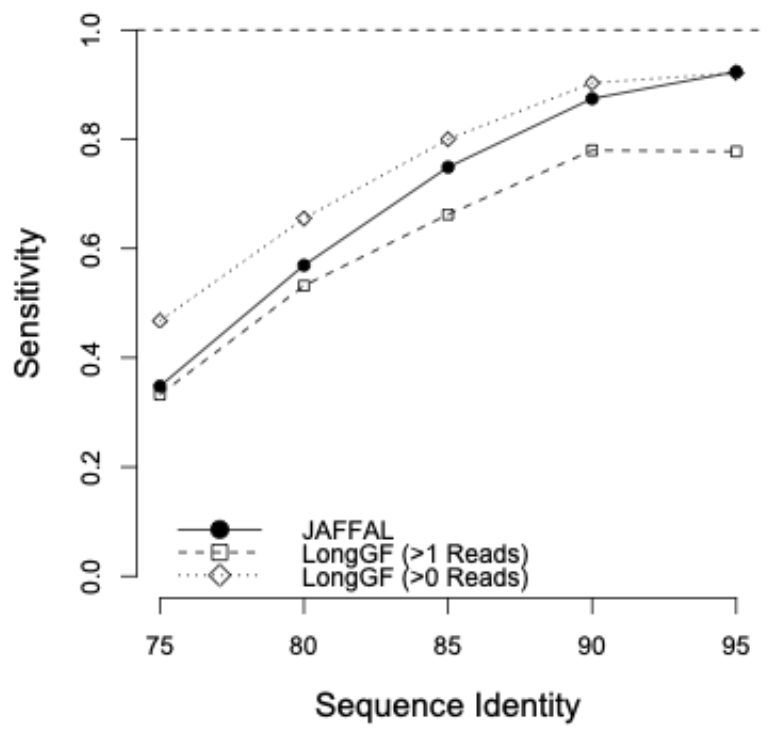

Figure 2: Fusion finding sensitivity on simulated ONT data. A) The fraction of simulated fusions detected (y-axis) by JAFFAL across a range of fusion coverage levels (x-axis). Read identity levels are shown in different colours (red-purple). B) The fraction of simulated fusions detected (y-axis) by JAFFAL and LongGF for sequence identity levels of $75-95 \%$. Results from PacBio simulation were similar (Supplementary Figure 2).

Although no background reads from unfused genes were generated, JAFFAL reported 118 and 129 false positives for the ONT and PacBio simulations respectively. False positives did not appear to be associated with simulated read identity. However most false positives, 81 for ONT and 97 for PacBio, had one breakpoint within 20bp of a simulated breakpoint, but the partner gene was misidentified, we presume due to sequence homology. LongGF (with $>0$ reads) reported fewer false positives than JAFFAL, 79 and 80 for the ONT and PacBio simulations respectively, and similarly the majority of those could be explained by one constituent gene 
being incorrectly identified (56 for ONT and 57 for PacBio). Overall both tools showed comparable performance on the simulated data.

JAFFAL's fusion ranking is effective at separating false positives in non-tumor cell line data

To assess the false positive rate of JAFFAL in more detail, we next applied it to deep ONT transcriptome sequencing of the reference cell line NA12878 where few fusions should be present. The data was generated by the Nanopore WGS consortium [32] and consisted of 94 million and 172 million reads respectively from direct RNA and amplified cDNA sequencing protocols. Raw data was base called with Guppy version 4.2.2.

For both protocols JAFFAL reported few fusions with a ranking of high confidence as expected (Table 1). Amongst the high confidence calls, three were common to both the direct RNA and cDNA datasets. One of these, KANSL1-ARL17A, is a germline fusion known to be present in a subset of the healthy population [33]. The two other fusions were consistent with run through transcription, where the distance between breakpoints just exceeded the 200kbp threshold for filtering. A further two fusions called in the cDNA sample could be explained as run through transcription for the same reason. JAFFAL reported several hundred "Potential Trans-splicing" events, which was consistent with levels seen previously from short read sequencing [23]. LongGF detected just five fusions with multi-read support for the direct RNA protocol, all of which were also reported by JAFFAL (two as high and three as low confidence).

\begin{tabular}{|c|c|c|c|}
\hline & & Direct RNA & cDNA \\
\hline \multicolumn{2}{|c|}{ Total Reads Processed } & $93,711,512$ & $172,247,744$ \\
\hline \multirow{3}{*}{$\begin{array}{l}\text { Fusions called by } \\
\text { JAFFAL }\end{array}$} & High Confidence & 4 & 8 \\
\hline & Low Confidence & 5 & 94 \\
\hline & $\begin{array}{l}\text { Potential } \\
\text { Trans-splicing }\end{array}$ & 344 & 412 \\
\hline \multirow{2}{*}{$\begin{array}{l}\text { Fusions called by } \\
\text { Long GF }\end{array}$} & > 1 Read support & 5 & 173 \\
\hline & $=1$ Read support & 713 & 386 \\
\hline
\end{tabular}


Table 1: The number of fusions called in the non-cancer cell line NA12878 from ONT direct RNA and amplified cDNA. Most calls are presumed to be false positives. The number of fusions in the highest rank category for each tool is shown in bold. We hypothesize that most of the multi-read fusions reported by LongGF applied to the cDNA dataset (173) are chimeras introduced during library preparation. JAFFAL ranks these events as Low Confidence.

However, on the cDNA data, LongGF reported 173 multi-read support fusions. We observed a similar difference in the number of low confidence calls from JAFFAL in the direct RNA (5 fusions reported) compared to the cDNA protocol (94 fusions reported) (Table 1). We hypothesise this is due to chimeric molecule creation during cDNA library preparation [34,35]. The ranking of these fusions as low confidence is consistent with this hypothesis because a hallmark of these events are breakpoints occurring within exons, rather than at exon boundaries and allows them to be separated from true fusions by JAFFAL's ranking. LongGF does not appear to separate this class of artifact and reported a large number of false positives in the cDNA dataset (Table 1).

To examine chimeras further, we switched off JAFFAL's default filtering of mitochondrial genes, and looked at the prevalence of fusions reported between a gene on the mitochondrial chromosome and a gene on another chromosome. These are likely to be chimeras which are not native to cells. 116 such mitocondrial chimeras were reported by JAFFAL in the CDNA library at low confidence. None were reported at other confidence levels or in the direct RNA library. To confirm this result in an independent dataset, we examined chimeras in data from five cell lines from the Singapore Nanopore-Expression Project, SGNex [36], where several replicates of direct RNA, direct cDNA and amplified cDNA ONT sequencing are available. A much lower rate of mitochondrial chimeras was seen in the direct RNA sequencing, but no significant difference was observed between direct and amplified cDNA (Supplementary Figure 4).

These results demonstrate that chimeras created during library preparation can be effectively separated from true fusions if fusion breakpoints are accurately determined and their position relative to exon boundaries used. The absence of chimeras in direct RNA sequencing is striking and gives confidence in the fusions called from this protocol. In particular, the filtering based on breaks occurring at the exon boundary can be removed allowing confident detection of the rare instances where a breakpoint occurs within an exon. 


\section{JAFFAL detects known fusions in cancer cell lines}

To further confirm JAFFAL's accuracy, it was applied to public long read transcriptome sequencing of six cancer cell line, where fusions were previously validated or there was orthogonal evidence of a translocation from whole genome sequencing [4,37-46] (Table 2, Supplementary Table 1). The four cell lines MCF-7, HCT-116, A549 and K562 were sequenced with ONT and are available as part of SGNex [36]. The direct RNA, direct cDNA and amplified cDNA replicates were combined into a single fastq file for fusion calling on each cell line. These samples had estimated read identities of $80-85 \%$ (Supplementary Figure 1). The three cell lines MCF-7, HCT-116 and SK-BR-3 [42] which had PacBio SMRT sequencing were downloaded from the Sequence Read Archive (SRA) and had estimated read identities over 95\% (MCF-7 and HCT-116) and $~ 86 \%$ (SK-BR-3).

JAFFAL rediscovered approximately half the previously validated fusions (Table 2) and $84 \%$ of these were ranked as high confidence. Compared to LongGF, JAFFAL reported equal or more previously validated fusions for all datasets and ranked them higher (Figure 3A and B, Table 2). JAFFAL also reported fewer total fusions in six of seven datasets, with unvalidated detections likely to be predominately false positives. These were reported by JAFFAL to be mainly in the potential trans splicing category similar to those seen in the reference cell line NA12878.

Although JAFFAL failed to report a number of the previously validated fusions, this could be caused by differences in sequencing depth and cell line batch effects, and is something that has been observed previously in short read data [23]. Hence, we also benchmarked JAFFAL's sensitivity against fusions called on matched short read data from the same samples. We used MCF-7 from SGNex, as this cell line had the greatest number of validated fusions. Fusions were called in the short read data with JAFFA, which has been independently benchmarked in several studies $[10,47,48]$. Because the long and short read sequencing were from the same replicates, we would expect a similar set of fusions to be expressed. The short read data was significantly more deeply sequenced (137 million 150bp paired-end Illumina reads), hence we subsampled the datasets to approximately $22 \mathrm{Gbp}, 4 \mathrm{Gbp}$, and $1 \mathrm{Gbp}$ to compare performance over a range of depths. The precision and recall of JAFFAL on long reads was found to be within the range of short read replicates, demonstrating both the accuracy of JAFFAL and the utility of noisy long read data for fusion detection more generally (Figure $3 \mathrm{C}$ ). 
The short read data of MCF-7 was also used to assess the likelihood of JAFFAL's unvalidated calls being genuine fusions. Genuine fusions should be found in both the ONT and full depth Illumina data. Of the 69 high confidence fusion genes called by JAFFAL, 60 were also detected in the short read data. 5 of 29 low confidence and 19 of 819 potential trans-splicing events were common, indicating that events in these categories are more likely to be artifacts, such as chimeras generated during library preparation, which is consistent with results from NA12878.

Breakpoint positions from JAFFAL were also consistent with short read data. For the 84 fusion genes common to the short and long read data across all confidence levels, 140 different breakpoints were reported by JAFFAL (range: 1-13 per fusion pair) and 181 by JAFFA (range: 1-15 per fusion pair) on the short read data. 117 of these were common between the short and long read datasets (within 20bp), with the majority, 104, an exact match. Note that the number of breakpoints is greater than the number of fusion genes likely due to alternative splicing. Correctly identifying all breakpoints is important for determining whether any fusion transcript is in-frame.

Overall on the MCF-7 ONT cell line data, JAFFAL's high and low confidence calls showed consistency with previously validated fusions, fusions in matched short read data and fusions called by LongGF (Figure 3D). Only $16 \%$ of fusions reported by JAFFAL were not seen by other approaches, compared to $70 \%$ of LongGF's calls. Taken together, these results suggest JAFFAL is highly accurate, in particular in the high confidence class. 


\begin{tabular}{|c|c|c|c|c|c|c|c|c|}
\hline & & $\begin{array}{l}\text { PacBio } \\
\text { HCT-116 }\end{array}$ & $\begin{array}{l}\text { PacBio } \\
\text { SK-BR-3 }\end{array}$ & $\begin{array}{l}\text { PacBio } \\
\text { MCF-7 }\end{array}$ & $\begin{array}{l}\text { ONT } \\
\text { HCT-116 }\end{array}$ & $\begin{array}{l}\text { ONT } \\
\text { A549 }\end{array}$ & $\begin{array}{l}\text { ONT } \\
\text { K562 }\end{array}$ & $\begin{array}{l}\text { ONT } \\
\text { MCF-7 }\end{array}$ \\
\hline \multicolumn{2}{|l|}{ Reads } & 156,632 & $3,070,545$ & $2,389,856$ & $44,416,838$ & $31,393,964$ & $36,751,242$ & $34,654,115$ \\
\hline \multicolumn{2}{|c|}{ \# Previously Validated Fusions } & 3 & 30 & 53 & 3 & 2 & 6 & 53 \\
\hline \multirow{4}{*}{$\begin{array}{l}\text { JAFFAL } \\
\text { \# Previously } \\
\text { Validated Fusions } \\
\text { Rediscovered } \\
\text { (All Fusions) }\end{array}$} & $\begin{array}{l}\text { High } \\
\text { Confidence }\end{array}$ & $1(1)$ & $13(20)$ & $26(73)$ & $3(49)$ & $2(21)$ & $2(17)$ & $29(69)$ \\
\hline & $\begin{array}{l}\text { Low } \\
\text { Confidence }\end{array}$ & $0(1)$ & $0(5)$ & $1(112)$ & $0(81)$ & $0(40)$ & $0(31)$ & $1(29)$ \\
\hline & $\begin{array}{l}\text { Potential } \\
\text { Trans-splicing }\end{array}$ & $0(21)$ & $1(201)$ & $9(435)$ & $1^{*}(2342)$ & $0(1206)$ & $0(615)$ & 2 (819) \\
\hline & Total & $1(23)$ & $14(226)$ & $36(620)$ & $4^{*}(2475)$ & 2 (1267) & $2(663)$ & 32 (917) \\
\hline \multirow{3}{*}{$\begin{array}{l}\text { LongGF } \\
\text { \# Previously } \\
\text { Validated Fusions } \\
\text { Rediscovered } \\
\text { (All Fusions) }\end{array}$} & $\begin{array}{l}>1 \text { Read } \\
\text { Support }\end{array}$ & $1(2)$ & $10(20)$ & $21(292)$ & $2(307)$ & $2(224)$ & $2(168)$ & $23(220)$ \\
\hline & $\begin{array}{l}=1 \text { Read } \\
\text { Support }\end{array}$ & $0(113)$ & $1(2537)$ & $6(1800)$ & $0(1321)$ & $0(1922)$ & $0(2267)$ & $3(2172)$ \\
\hline & Total & $1(115)$ & 11 (2557) & 27 (2092) & $2(1628)$ & $2(2146)$ & $2(2435)$ & 26 (2392) \\
\hline
\end{tabular}

Table 2: The number of previously validated fusions rediscovered across seven long read sequencing datasets by JAFFAL and LongGF. The total number of fusions reported by each tool, including those not previously validated, are indicated in parentheses. * As LongGF does not report fusion partners in order, reciprocal translocations were counted as validated. JAFFAL reported COMMD10-AP3S1 and AP3S1-COMMD10 in the ONT sequencing of HCT-116. 

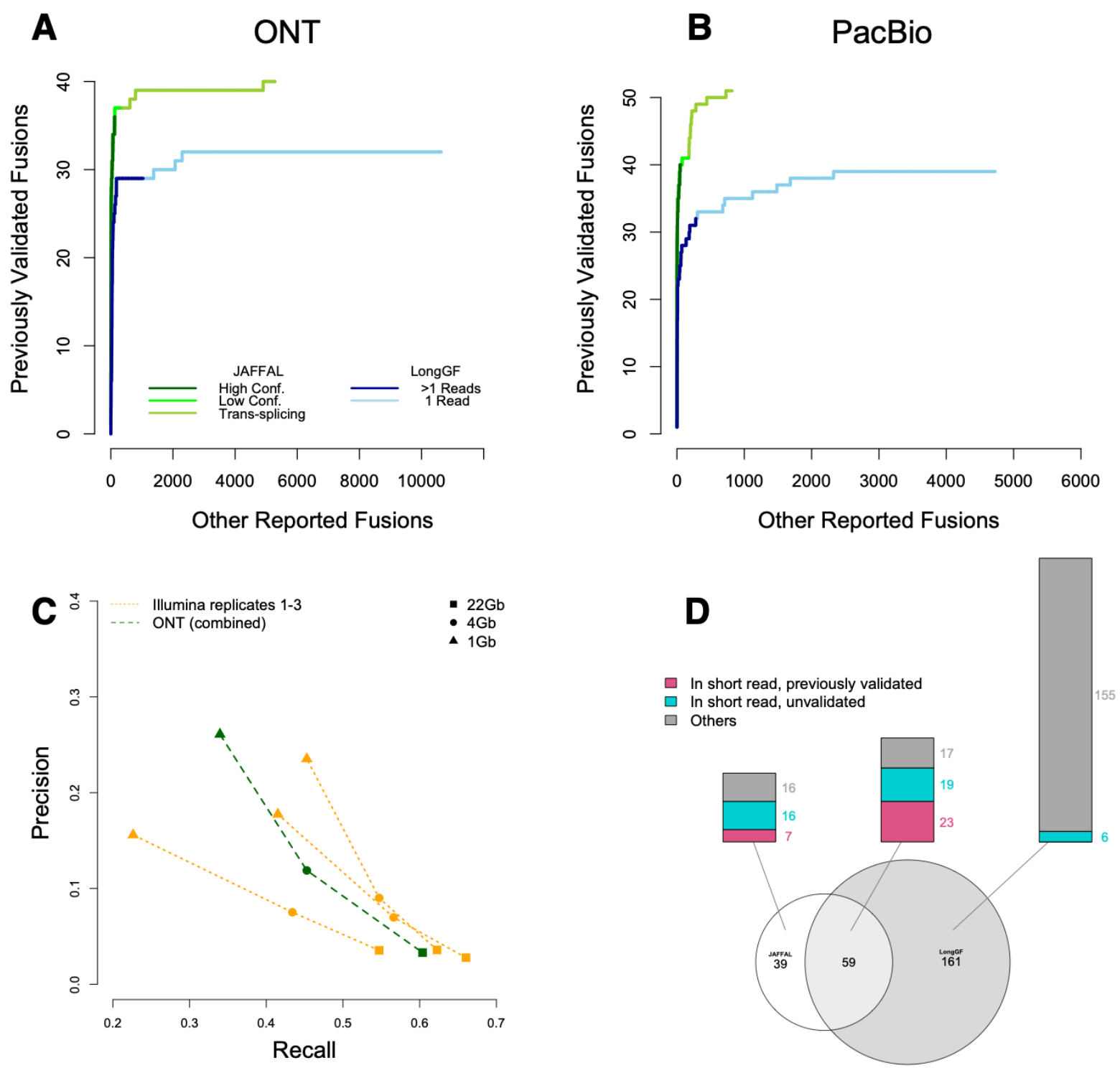

Figure 3: Comparison of JAFFAL and LongGF on cancer cell line sequencing. Shown are ROC style curve with the ranking of previously validated fusions against other reported fusions for A) MCF-7, HCT-116, A549 and K562 cell lines sequenced with ONT and B) MCF-7, HCT-116 and SK-BR-3 cell lines sequenced with PacBio. C) For MCF-7 only, fusions from JAFFAL are compared against three short read Illumina replicates across three sequencing depths. Precision and recall are calculated using previously validated fusions in MCF-7. D) The overlap between fusions called by JAFFAL (high and low confidence) and LongGF (>1 read support) on MCF-7. 


\section{Detection of clinically relevant fusions with long read sequencing in leukemia}

JAFFAL was next applied to two samples from patients with leukemia to assess its ability to detect fusions in a real-word context. One patient had acute myeloid leukemia (AML) with a RUNX1-RUNX1T1 fusion and cDNA sequencing was performed by Lui et al. [25] on ONT GridION, resulting in 8 million reads. The other patient had B-cell acute lymphoblastic leukemia (B-ALL) with the rare phenomenon of both BCR-ABL1 and IGH-CRLF2 fusions detected by cytogenetics and short read RNA sequencing. ONT sequencing was performed on amplified cDNA with a MinION, resulting in 13 million reads.

JAFFAL detected the RUNX1-RUNX1T1 and BCR-ABL1 fusions ranked as first and fifth in their respective samples, both as high confidence. Consistent with results from simulation and cell line data, JAFFAL found the exact breakpoints. However it failed to detect the IGH-CRLF2 fusion, despite the fusion transcript being evident through manual inspection in the sequencing data. IGH-CRLF2 was missed because the breakpoint occurred approximately $2 \mathrm{kbp}$ upstream of CRLF2 and is an example of enhancer hijacking. Inability to detect fusions involving intergenic regions is an important limitation of JAFFAL, but is one shared by most fusion finders, with a few exceptions [9,49]. LongGF also failed to detect the IGH-CRLF2 fusion (Supplementary Table 2).

\section{Fusion detection at the single cell level}

Single cell transcriptomics using long read sequencing is emerging as a powerful system to investigate transcript diversity across cell types [18-20]. As tumour samples nearly always contain multiple cell types, including infiltration of immune cells [50], or multiple clones [51], it is of broad interest to track the presence of fusion genes within single cells. As a proof of the feasibility for calling fusions at the single cell level, we applied JAFFAL to public data from a mixed sample of five cancer cell lines that was sequenced with ONT in combination with 10x Genomics and Illumina sequencing [19]. A total of 18 million ONT reads could be assigned cellular barcodes across 557 cells. As expected, cells clustered into five distinct groups based on gene expression from short read data (Figure 4A). High confidence fusions called by JAFFAL in four or more cells were investigated further. JAFFAL identified 16 fusions, 12 of which were also found in short read RNA-seq of the same cell lines as part of the Cancer Cell Line Encyclopedia, CCLE [52] (Figure 4B). Distinct sets of fusions were associated with each cluster, 
enabling the annotation of the cluster to each of the cell lines (Figure 4A). One fusion, RP11-96H19.1-RP11-446N19.1, was seen across all five clusters. It is not present in CCLE and is consistent with run-through transcription with constituent genes $264 \mathrm{kbp}$ apart in the reference genome (Figure 4B). For some cells, fusions were inconsistent with gene expression clustering and we hypothesize that long read sequencing errors in the cell barcodes have led to misassignment of reads in these cases. However, despite errors, these results demonstrate that JAFFAL enables fusions to be detected at the level of individual cells.

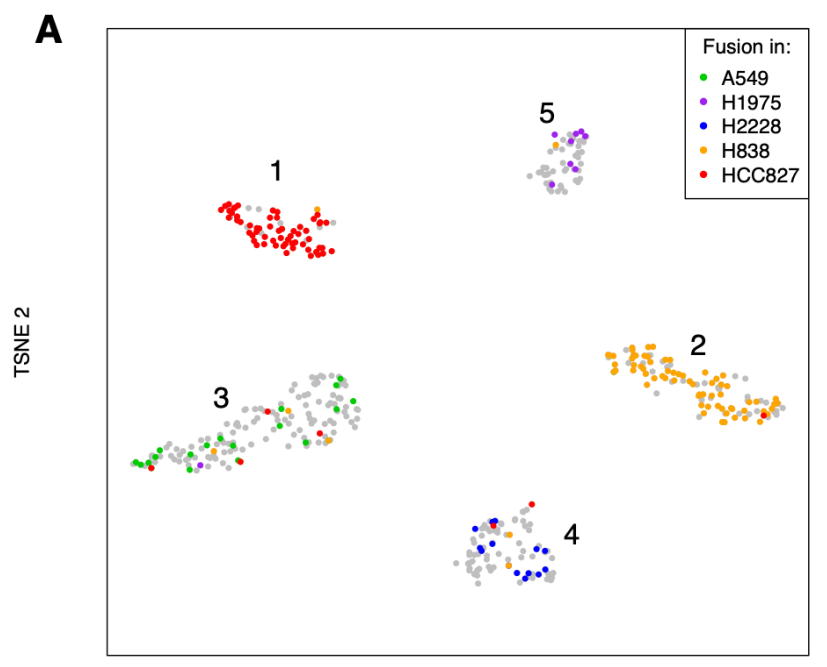

TSNE 1

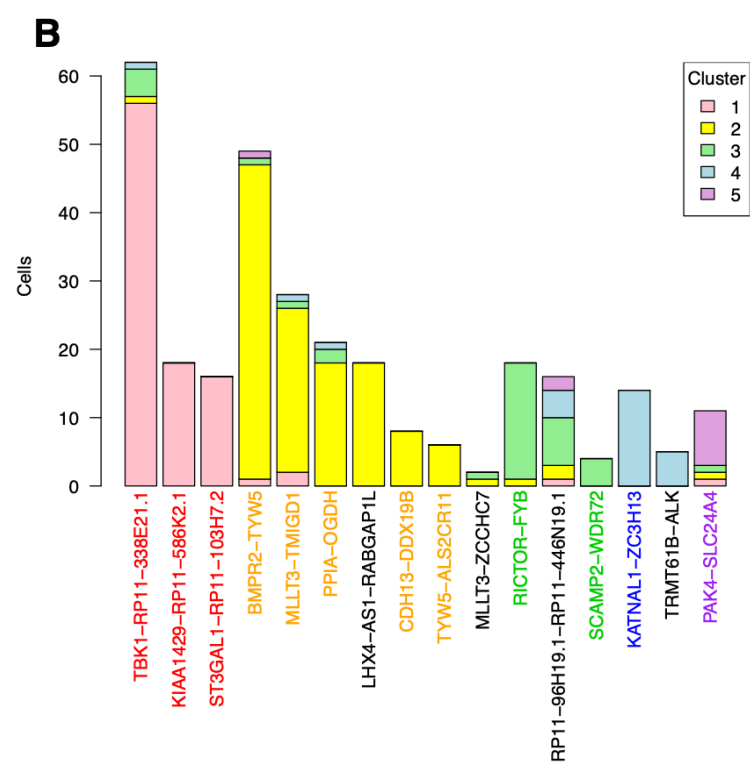

Figure 4: Detection of fusions in single cell ONT sequencing of five cell lines. A) t-SNE plot generated from short read gene expression. Colour indicates the cell line that a fusion detection is known to be in from CCLE. Grey indicates a cell with no detected CCLE fusion. B) For each of the 16 fusions detected by JAFFAL the number of cells identified, in each of the five clusters is shown. Fusion labels are coloured according to the CCLE cell line they were previously identified in. Black indicates a novel fusion.

\section{Computational Resources}

The computational resources required for JAFFAL and LongGF were benchmarked on a machine with 32 cores and 190GB of available memory. All tools were given a maximum of four threads, apart from LongGF which is single threaded. JAFFAL completed in less than six hours and $21 \mathrm{~GB}$ of memory on each of the nine healthy and cancer cell line bulk datasets described 
previously (Table 4). LongGF used less computational resources, but required reads which had already been mapped to the genome. Genome alignment using minimap2 was slower than JAFFAL, but required approximately the same memory. These results indicate that fusion calling on large long read sequencing cohorts is unlikely to be hindered by computational limitations using either fusion finder.

\begin{tabular}{|c|c|c|c|}
\hline & & Run-time (hours) & $\begin{array}{l}\text { Memory Consumption } \\
\text { (GB) }\end{array}$ \\
\hline \multicolumn{2}{|c|}{ JAFFAL (4 threads) } & $2.6(0.08-5.9)$ & $20.0(19.8-21.1)$ \\
\hline \multirow[t]{2}{*}{ LongGF } & Genome Mapping (4 threads) & $9.5(0.1-21.2)$ & $22.6(20.2-24.7)$ \\
\hline & LongGF (1 thread) & $0.4(0.01-1.1)$ & $6.4(0.8-13.3)$ \\
\hline
\end{tabular}

Table 4: Average and range (in parentheses) of run-time and memory consumed on nine benchmarking datasets by JAFFAL and LongGF.

\section{Conclusions}

Long read sequencing is growing in popularity due to its ability to measure long stretches of sequence. A natural application is therefore detecting structural rearrangements, which in the transcriptome, can arise as fusions. However, very few computational methods exist for fusion detection from long read transcriptome sequencing. Here we introduce JAFFAL which is one of the first long read fusion finders. We demonstrated that JAFFAL is sensitive on simulated data over a range of read identities and coverage levels designed to mimic real ONT and PacBio data. On real data, JAFFAL detected previously known fusions in cancer cell lines and patient samples with few false positives.

The ranking of fusions for prioritisation is an important feature of fusion finders. While alternative methods rely on the number of read support only, we demonstrate that other heuristics are powerful for separating artifacts from true fusions. By applying JAFFAL to samples sequenced with both direct RNA and CDNA we found a high rate of chimeric artifacts introduced during reverse transcription of libraries. We showed that these can be controlled by either sequencing RNA directly or by downranking fusions if their breakpoint does not coincide with exon boundaries. 
Although the idea of using breakpoint positions as a heuristic in fusion ranking was first introduced for short read data [23], errors in long reads make precise breakpoints difficult to determine. JAFFAL overcomes this challenge by clustering reads into breakpoints and anchoring them to exon boundaries or the position with the maximum read support, per cluster. This approach is one clear advantage of JAFFAL and was found to give fewer false positives compared to the competing long read tool, LongGF on cancer cell line data.

JAFFAL and LongGF were found to identify different fusions when applied to the MCF-7 cell line. Differences between fusions called by different tools on short read data are well documented, and this is why clinical pipelines often employ an ensemble approach combining the results of several fusion finding tools together, to identify actionable fusions $[5,48,53]$. It is likely that long read fusion finding will also benefit from multiple methods being available, and JAFFAL represents an important early contribution towards this.

A limitation of JAFFAL is its dependence on annotated transcripts. Fusions which incorporate intergenic or intronic sequences at a breakpoint are not detected. Hence complex fusions such as IGH-CRLF2 in our patient sample will be missed. This highlights an area for further development in long read fusion finding. As shown in our simulation, the detection of fusions is limited by their coverage, which is directly related to expression levels, and by error rates in the data. However, sequencing accuracy from long read technologies is improving and is likely to benefit fusion finding with JAFFAL in the future.

Finally, long read sequencing has a number of novel advantages over short reads. An exciting development has been the use of long reads in conjunction with single cell RNA sequencing, which enables the full transcriptomes of individual cells to be sequenced. Here we demonstrate that fusions can be called in this data, adding an extra modality to single cell analysis, providing many new opportunities to study the heterogeneity of tumours. ONT sequencing has several further advantages including the profiling of the epitranscriptome and rapid and remote sequencing. Combined with fusion finding, these technological advances have the potential to enable greater understanding of the mechanisms driving tumours and the potential to bring clinical diagnostics to remote areas. 


\section{Materials and Methods}

\section{JAFFAL pipeline}

JAFFAL is a multi-stage bpipe [26] pipeline for fusion detection. A brief outline of its steps follow. Fastq files are unzipped and converted to fasta prior to alignment to the human reference transcriptome, gencode version 22 for hg38, with minimap2 version 2.17 and option $-x$ map-ont. Alignments to the transcriptome are then processed with a custom $\mathrm{C}++$ program, which identifies reads which align to two distinct genes. The two alignment intervals within a read must have no more than $15 \mathrm{bp}$ of overlap, no more than a $15 \mathrm{bp}$ gap and be on the same strand. Fusion candidate reads are then extracted into a fasta file and aligned to the reference genome, hg38 using minimap2 with option -x splice. Genome alignments are processed using a custom $\mathrm{R}$ script. It first finds the breakpoint positions in the genome and filters any where the start and end are within $10 \mathrm{kbp}$ of each other in an order consistent with regular transcription. Next alignments are compared against annotated transcripts, and breakpoints realigned to exon boundaries as described in Results. Fusions involving the mitochondrial chromosome are filtered out (by default). Reads are then aggregated by breakpoint, and clustered using the following algorithm:

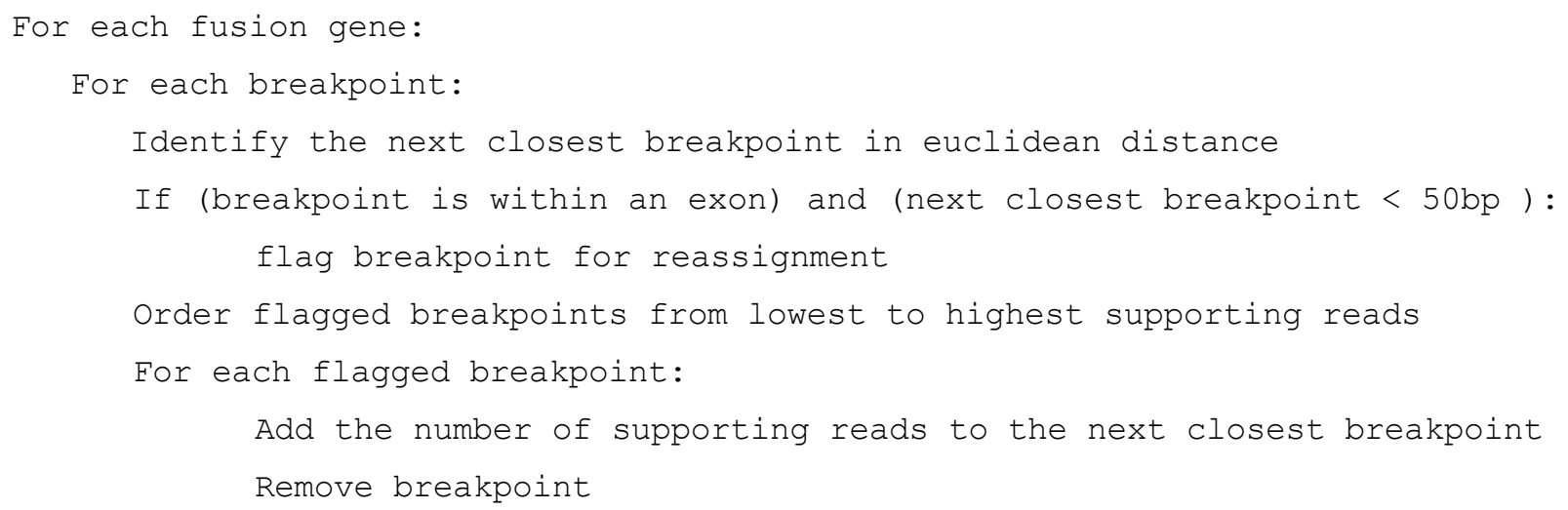

Next, breakpoints are classified as either high confidence, low confidence, potential trans-splicing (Figure 1) or run-through transcription. Run-through transcription includes any fusion with breakpoints that are within $200 \mathrm{kbp}$ of each other in an order consistent with regular transcription and these are filtered out by default. Information on whether the fusion is in frame and seen in the Mitelman database is added. Final breakpoints are reported in a csv output file. The code for JAFFAL is open source and available at https://github.com/Oshlack/JAFFA. The results presented in this manuscript were generated with JAFFAL version 2.1 run with the flag $-n 4$ (4 threads). 


\section{LongGF}

Samples were first mapped to version hg38 of the human reference genome using minimap2 version 2.17 with flags - $t 4$ and -ax splice. Mapped reads were name sorted with samtools before being processed with LongGF version 0.1.1. We ran LongGF with the annotation file gencode.v22.chr_patch_hapl_scaff.annotation.gtf downloaded from https://www.gencodegenes.org/human/release_22.html. We used options "100 5010000 1" which were recommended apart from the number of reads support which we lowered from 2 to 1 to assess sensitivity. Fusions involving a gene on the mitochondrial chromosome were removed to allow consistent comparison against JAFFAL which removes these by default.

\section{Simulation}

Simulated fusion transcripts created by Haas et al. [10] were downloaded and split into 25 fasta files for each of the 25 combinations of coverage-levels $(1,2,10,50,100)$ and read identities $(75 \%, 80 \%, 85 \%, 90 \%, 95 \%)$. Sequencing reads were then simulated using Badread version

0.1 .5 with corresponding coverage and read identity levels set through the parameters --quantity $<$ coverage> and --identity <read identity>,95,5 respectively. The error model was set to either pacbio or nanopore with the parameters --error_model and --qscore_model. To simplify the simulation, we switched off artifacts with the options --junk_reads 0 --random_reads 0 --chimeras 0 . Chimeras in long reads were assessed with real data rather than simulation.

\section{Comparison}

For the simulation, fusions were classed as true positives if there was a simulated breakpoint within $1 \mathrm{kbp}$ euclidean distance of the reported breakpoint. All other fusions were considered false positives. For cell lines data we matched fusions to those previously validated (Supplementary Table 1) and between fusion finders using gene identifiers. As LongGF does not report fusion genes in transcriptional order, we also checked for the reciprocal fusion and counted these as a match if found. Unless stated otherwise, comparisons were performed at the fusion gene-level, meaning fusions with multiple breakpoints were counted as a single true or false positive, and given the ranking of their highest ranked breakpoint.

\section{Patient Sequencing}

For the B-ALL patient sample, an ONT sequencing library was generated from approximately $100 \mathrm{ng}$ of total RNA using the ONT cDNA-PCR Sequencing Kit (SQK-PCS109) and sequenced 
using a MinION Nanopore sequencer on a R9.4 flow cell (FLO-MIN106). Basecalling was performed using Guppy version 4.2.3.

\section{Single cell analysis}

Cellular barcodes were annotated to long reads using FLAMES [19]. JAFFAL was then run on pooled reads. A custom script, get_cell_barcodes_by_fusion.bash, which is available in JAFFA, was used to generate a table of fusions by cell barcode. Only fusions classes as high confidence and found in four or more cells were analysed further. Matched short read gene expression count data from Tian et al. [19] was downloaded from https://github.com/LuyiTian/FLTseq_data/blob/master/data/PromethION_scmixology1.zip and analysed with Seurat [54]. A list of fusions called in short read CCLE data was obtained from the CCLE data portal, https://portals.broadinstitute.org/ccle.

\section{Abbreviations}

ONT - Oxford Nanopore Technologies

ALL - acute lymphoblastic leukemia

AML - acute myeloid leukemia

SRA - Sequence Read Archive

CCLE - Cancer Cell Line Encyclopedia

\section{Declarations}

\section{Ethics approval and consent to participate}

ONT sequencing of the B-ALL patient sample was conducted under Peter MacCallum Cancer Centre Ethics Committee approval (HREC/17/PMCC/163) and all experiments were performed in accordance with the Declaration of Helsinki.

\section{Consent for publication}

Not applicable

\section{Data Availability}

Simulated data is available for download from figshare [55]. NA12878 cell line data was downloaded from https://github.com/nanopore-wgs-consortium/NA12878. SGNex ONT cell line sequencing is available at https://github.com/GoekeLab/sg-nex-data. PacBio sequencing of 
MCF-7, HCT-116 and SK-BR-3 cell lines are available from the sequence read archive (SRA) under accessions SRP055913, SRP091981 and SRP150606 respectively. The patient sample from Lui et al. [25] is available from SRA under accession SRP267910. The long read data for the B-ALL patient sample is available from the corresponding authors on reasonable request. The ONT single cell sequencing is available under SRA accession SRP273167.

\section{Competing interests}

The authors declare that they have no competing interests

\section{Funding}

NMD and AO are funded by NHMRC project grant GNT1140626. AO is supported by NHMRC Investigator Grant GNT1196256.

\section{Authors' contributions}

NMD and AO conceived of ideas in this manuscript and designed the research. NMD wrote the software, performed all the analysis and wrote the preliminary draft of the manuscript. JG and YC provided cancer cell line data and contributed to concept development. GLR and PB provided patient data. NMD, GLR, PB, JG and AO revised the manuscript. All authors read and approved the final manuscript.

\section{Acknowledgements}

The authors would like to thank Mike Clark and Ricardo De Paoli-Iseppi (Centre for Stem Cell Systems, Department of Anatomy and Neuroscience, The University of Melbourne) for their assistance with experimental design, library preparation and sequencing of patient samples. We would also like to thank Matt Ritchie and Luyi Tian for access to long read single cell datasets. Computing resources for this work were provided by PeterMac and The University of Melbourne Science IT.

\section{References}

1. Mitelman F, Johansson B, Mertens F. The impact of translocations and gene fusions on cancer causation. Nat Rev Cancer. Nature Publishing Group; 2007;7:233-45.

2. Druker BJ, Talpaz M, Resta DJ, Peng B, Buchdunger E, Ford JM, et al. Efficacy and safety of a specific inhibitor of the BCR-ABL tyrosine kinase in chronic myeloid leukemia. N Engl J Med. 
2001;344:1031-7.

3. Maher CA, Kumar-Sinha C, Cao X, Kalyana-Sundaram S, Han B, Jing X, et al. Transcriptome sequencing to detect gene fusions in cancer. Nature. 2009;458:97-101.

4. Edgren H, Murumagi A, Kangaspeska S, Nicorici D, Hongisto V, Kleivi K, et al. Identification of fusion genes in breast cancer by paired-end RNA-sequencing. Genome Biol. 2011;12:R6.

5. Wong M, Mayoh C, Lau LMS, Khuong-Quang D-A, Pinese M, Kumar A, et al. Whole genome, transcriptome and methylome profiling enhances actionable target discovery in high-risk pediatric cancer. Nat Med [Internet]. 2020; Available from:

https://doi.org/10.1038/s41591-020-1072-4

6. Gao Q, Liang W-W, Foltz SM, Mutharasu G, Jayasinghe RG, Cao S, et al. Driver Fusions and Their Implications in the Development and Treatment of Human Cancers. Cell Rep. 2018;23:227-38.e3.

7. McPherson A, Hormozdiari F, Zayed A, Giuliany R, Ha G, Sun MGF, et al. deFuse: an algorithm for gene fusion discovery in tumor RNA-Seq data. Markel S, editor. PLoS Comput Biol. Public Library of Science; 2011;7:e1001138.

8. Kim D, Salzberg SL. TopHat-Fusion: an algorithm for discovery of novel fusion transcripts. Genome Biol. 2011;12:R72.

9. Uhrig S, Ellermann J, Walther T, Burkhardt P, Fröhlich M, Hutter B, et al. Accurate and efficient detection of gene fusions from RNA sequencing data. Genome Res [Internet]. 2021; Available from: http://genome.cshlp.org/content/early/2021/01/13/gr.257246.119.abstract

10. Haas BJ, Dobin A, Li B, Stransky N, Pochet N, Regev A. Accuracy assessment of fusion transcript detection via read-mapping and de novo fusion transcript assembly-based methods. Genome Biol. 2019;20:213.

11. Jia W, Qiu K, He M, Song P, Zhou Q, Zhou F, et al. SOAPfuse: an algorithm for identifying fusion transcripts from paired-end RNA-Seq data. Genome Biol. 2013;14:R12.

12. Melsted P, Hateley S, Joseph IC, Pimentel H, Bray N, Pachter L. Fusion detection and quantification by pseudoalignment [Internet]. Cold Spring Harbor Laboratory. 2017 [cited 2021 Feb 16]. p. 166322. Available from: https://www.biorxiv.org/content/10.1101/166322v1

13. Clarke J, Wu H-C, Jayasinghe L, Patel A, Reid S, Bayley $\mathrm{H}$. Continuous base identification for single-molecule nanopore DNA sequencing. Nat Nanotechnol. 2009;4:265-70.

14. Eid J, Fehr A, Gray J, Luong K, Lyle J, Otto G, et al. Real-time DNA sequencing from single polymerase molecules. Science. 2009;323:133-8.

15. Liu H, Begik O, Lucas MC, Ramirez JM, Mason CE, Wiener D, et al. Accurate detection of m6A RNA modifications in native RNA sequences. Nat Commun. 2019;10:4079.

16. Jeck WR, lafrate AJ, Nardi V. Nanopore Flongle Sequencing as a Rapid, Single Specimen Clinical Test for Fusion Detection. J Mol Diagn [Internet]. 2021; Available from: http://dx.doi.org/10.1016/j.jmoldx.2021.02.001 
Assay for Rapid Detection of Gene Fusions. J Mol Diagn. 2019;21:58-69.

18. Singh M, Al-Eryani G, Carswell S, Ferguson JM, Blackburn J, Barton K, et al. High-throughput targeted long-read single cell sequencing reveals the clonal and transcriptional landscape of lymphocytes. Nat Commun. 2019;10:3120.

19. Tian L, Jabbari JS, Thijssen R, Gouil Q, Amarasinghe SL, Kariyawasam H, et al. Comprehensive characterization of single cell full-length isoforms in human and mouse with long-read sequencing [Internet]. 2020 [cited 2020 Aug 18]. p. 2020.08.10.243543. Available from: https://www.biorxiv.org/content/10.1101/2020.08.10.243543v1

20. Gupta I, Collier PG, Haase B, Mahfouz A, Joglekar A, Floyd T, et al. Single-cell isoform RNA sequencing characterizes isoforms in thousands of cerebellar cells. Nat Biotechnol [Internet]. 2018; Available from: http://dx.doi.org/10.1038/nbt.4259

21. Križanovic K, Echchiki A, Roux J, Šikic M. Evaluation of tools for long read RNA-seq splice-aware alignment. Bioinformatics. 2018;34:748-54.

22. Rang FJ, Kloosterman WP, de Ridder J. From squiggle to basepair: computational approaches for improving nanopore sequencing read accuracy. Genome Biol. 2018;19:90.

23. Davidson NM, Majewski IJ, Oshlack A. JAFFA: High sensitivity transcriptome-focused fusion gene detection. Genome Med. 2015;7:43.

24. Rautiainen M, Durai DA, Chen Y, Xin L, Low HM, Göke J, et al. AERON: Transcript quantification and gene-fusion detection using long reads [Internet]. bioRxiv. 2020 [cited 2020 Feb 5]. p. 2020.01.27.921338. Available from: https://www.biorxiv.org/content/10.1101/2020.01.27.921338v1

25. Liu Q, Hu Y, Stucky A, Fang L, Zhong JF, Wang K. LongGF: computational algorithm and software tool for fast and accurate detection of gene fusions by long-read transcriptome sequencing. BMC Genomics. 2020;21:793.

26. Sadedin SP, Pope B, Oshlack A. Bpipe: a tool for running and managing bioinformatics pipelines. Bioinformatics. 2012;28:1525-6.

27. Harrow J, Frankish A, Gonzalez JM, Tapanari E, Diekhans M, Kokocinski F, et al. GENCODE: the reference human genome annotation for The ENCODE Project. Genome Res. 2012;22:1760-74.

28. Li H. Minimap2: pairwise alignment for nucleotide sequences. Bioinformatics. 2018;34:3094-100.

29. Lei Q, Li C, Zuo Z, Huang C, Cheng H, Zhou R. Evolutionary Insights into RNA trans-Splicing in Vertebrates. Genome Biol Evol. 2016;8:562-77.

30. Mitelman F, Johansson B, Mertens F. Mitelman Database of Chromosome Aberrations and Gene Fusions in Cancer [Internet]. 2014. Available from:

http://cgap.nci.nih.gov/Chromosomes/Mitelman

31. Wick R. Badread: simulation of error-prone long reads. J Open Source Softw. The Open Journal; 2019;4:1316. 
32. Workman RE, Tang AD, Tang PS, Jain M, Tyson JR, Razaghi R, et al. Nanopore native RNA sequencing of a human poly(A) transcriptome. Nat Methods. 2019;16:1297-305.

33. Zhou JX, Yang X, Ning S, Wang L, Wang K, Zhang Y, et al. Identification of KANSARL as the first cancer predisposition fusion gene specific to the population of European ancestry origin. Oncotarget. 2017;8:50594-607.

34. White R, Pellefigues C, Ronchese F, Lamiable O, Eccles D. Investigation of chimeric reads using the MinION. F1000Res. 2017;6:631.

35. Houseley J, Tollervey D. Apparent Non-Canonical Trans-Splicing Is Generated by Reverse Transcriptase In Vitro. PLoS One. Public Library of Science; 2010;5:e12271.

36. Chen Y, Davidson N, Wan YK, Patel H, Yao F, Low HM, et al. A systematic benchmark of Nanopore long read RNA sequencing for transcript level analysis in human cell lines [Internet]. bioRxiv. 2021 [cited 2021 Apr 23]. p. 2021.04.21.440736. Available from:

https://www.biorxiv.org/content/10.1101/2021.04.21.440736v1

37. Kangaspeska S, Hultsch S, Edgren H, Nicorici D, Murumägi A, Kallioniemi O. Reanalysis of RNA-sequencing data reveals several additional fusion genes with multiple isoforms. Shipley J, editor. PLoS One. Public Library of Science; 2012;7:e48745.

38. Sakarya O, Breu H, Radovich M, Chen Y, Wang YN, Barbacioru C, et al. RNA-Seq mapping and detection of gene fusions with a suffix array algorithm. Zhang W, editor. PLoS Comput Biol. Public Library of Science; 2012;8:e1002464.

39. Maher CA, Palanisamy N, Brenner JC, Cao X, Kalyana-Sundaram S, Luo S, et al. Chimeric transcript discovery by paired-end transcriptome sequencing. Proc Natl Acad Sci U S A. 2009;106:12353-8.

40. Inaki K, Hillmer AM, Ukil L, Yao F, Woo XY, Vardy LA, et al. Transcriptional consequences of genomic structural aberrations in breast cancer. Genome Res. 2011;21:676-87.

41. Francis RW, Thompson-Wicking K, Carter KW, Anderson D, Kees UR, Beesley AH.

FusionFinder: a software tool to identify expressed gene fusion candidates from RNA-Seq data. PLoS One. 2012;7:e39987.

42. Nattestad M, Goodwin S, Ng K, Baslan T, Sedlazeck FJ, Rescheneder P, et al. Complex rearrangements and oncogene amplifications revealed by long-read DNA and RNA sequencing of a breast cancer cell line. Genome Res. 2018;28:1126-35.

43. Nome T, Hoff AM, Bakken AC, Rognum TO, Nesbakken A, Skotheim RI. High frequency of fusion transcripts involving TCF7L2 in colorectal cancer: novel fusion partner and splice variants. PLoS One. 2014;9:e91264.

44. Nome T, Thomassen GO, Bruun J, Ahlquist T, Bakken AC, Hoff AM, et al. Common fusion transcripts identified in colorectal cancer cell lines by high-throughput RNA sequencing. Transl Oncol. 2013;6:546-53.

45. Vellichirammal NN, Albahrani A, Banwait JK, Mishra NK, Li Y, Roychoudhury S, et al. Pan-Cancer Analysis Reveals the Diverse Landscape of Novel Sense and Antisense Fusion Transcripts. Mol Ther Nucleic Acids. 2020;19:1379-98. 
46. Asmann YW, Hossain A, Necela BM, Middha S, Kalari KR, Sun Z, et al. A novel bioinformatics pipeline for identification and characterization of fusion transcripts in breast cancer and normal cell lines. Nucleic Acids Res. 2011;39:e100.

47. Kumar S, Vo AD, Qin F, Li H. Comparative assessment of methods for the fusion transcripts detection from RNA-Seq data. Sci Rep. 2016;6:21597.

48. Liu S, Tsai W-H, Ding Y, Chen R, Fang Z, Huo Z, et al. Comprehensive evaluation of fusion transcript detection algorithms and a meta-caller to combine top performing methods in paired-end RNA-seq data. Nucleic Acids Res. 2016;44:e47.

49. Cmero M, Schmidt B, Majewski IJ, Ekert PG, Oshlack A, Davidson NM. MINTIE: identifying novel structural and splice variants in transcriptomes using RNA-seq data. bioRvix. 2020;2020.

50. Azizi E, Carr AJ, Plitas G, Cornish AE, Konopacki C, Prabhakaran S, et al. Single-Cell Map of Diverse Immune Phenotypes in the Breast Tumor Microenvironment. Cell.

2018;174:1293-308.e36.

51. Gerlinger M, Rowan AJ, Horswell S, Math M, Larkin J, Endesfelder D, et al. Intratumor heterogeneity and branched evolution revealed by multiregion sequencing. $\mathrm{N}$ Engl $\mathrm{J}$ Med. 2012;366:883-92.

52. Barretina J, Caponigro G, Stransky N, Venkatesan K, Margolin AA, Kim S, et al. The Cancer Cell Line Encyclopedia enables predictive modelling of anticancer drug sensitivity. Nature. 2012;483:603-7.

53. LaHaye S, Fitch JR, Voytovich KJ, Herman AC, Kelly BJ, Lammi GE, et al. Discovery of Clinically Relevant Fusions in Pediatric Cancer [Internet]. bioRxiv. 2021 [cited 2021 Apr 25]. p. 2021.03.11.435013. Available from:

https://www.biorxiv.org/content/10.1101/2021.03.11.435013v1.abstract

54. Stuart T, Butler A, Hoffman P, Hafemeister C, Papalexi E, Mauck WM 3rd, et al. Comprehensive Integration of Single-Cell Data. Cell. 2019;177:1888-902.e21.

55. Davidson N. Long Read Fusion Simulation [Internet]. 2021. Available from: https://figshare.com/articles/dataset/Long_Read_Fusion_Simulation/14459007 